\title{
Information Freshness in Random Access Channels for IoT Systems
}

\author{
Andrea Munari, Elif Uysal
}

\begin{abstract}
This paper introduces and discusses some recent advances in the field of information freshness for Internet of things (IoT) applications. Focusing on a setup in which a large number of transmitters share a common wireless channel to deliver updates to a common receiver, we tackle in particular the role played by random access policies based on variations of the basic ALOHA protocol, employed in most practical IoT systems. The fundamental trade-offs that emerge are discussed together with the enhancement achieved by a number of novel schemes tailored to optimize age of information. The article aims to trigger discussion and further research on a relevant yet still largely unexplored field, and highlights some important issues and challenges that have yet to be addressed.
\end{abstract}

\section{INTRODUCTION}

$\mathbf{R}$ ECENT years have witnessed a steadily growing interest towards the Internet of things (IoT), which is emerging as a core component in the design of next-generation wireless systems. Instantiated by a large number of applications, ranging from smart agriculture and industry to asset tracking and environmental monitoring, IoT communications are already supported by commercial products and standards. In this perspective, consolidated solutions in the terrestrial domain, e.g. LoRa, SigFox, NB-IoT [1]-[3], are being flanked by the possibility to provide connectivity via non-terrestrial networks (NTNs) (e.g., low-earth orbit satellites) [4], further broadening the potential of this paradigm.

IoT settings are typically characterized by the presence of a very large number of terminals, often with power and complexity constraints, that generate and transmit information addressed to a gateway in a sporadic and unpredictable fashion, e.g., related to the sampling of a physical process. The key goal of such networks is thus to maintain an upto-date perception at the receiver of the status of the monitored sources, as epitomized by cyber-physical systems, where control decisions made based on stale information may be detrimental. From this standpoint, performance criteria that are commonly employed for system design and optimization, e.g. throughput, packet delivery latency, or reliability, are not adequate to capture the core trade-offs of interest, as they focus on the channel utilization efficiency without regard to the freshness or importance of the information content being delivered.

To close this gap, recent research has focused on the definition of new relevant metrics. Among them, a pioneering role has been played by the notion of age of information (AoI)

A. Munari is with the Institute of Communications and Navigation, German Aerospace Center (DLR), 82234 Wessling, Germany (email: andrea.munari@dlr.de)

E. Uysal is with the Department of Electrical and Electronics Engineering, Middle East Technical University (METU), 06800 Ankara, Turkey (e-mail: uelif@metu.edu.tr).
[5], which tracks the time elapsed since the time-stamp of the newest successfully received update from a monitored source. Thanks to its simple definition, AoI has allowed to identify many novel and relevant trends, and a good level of maturity in its understanding has been reached in point-to-point settings, see e.g. [6] for an excellent survey.

While insightful, however, these results do not shed complete light on the fundamental limits that may characterize IoT settings, as the presence of multiple transmitters that contend for a shared channel triggers specific link layer issues that do not emerge in single-source/single-destination setups. This aspect becomes especially relevant when service has to be provided to a massive number of terminals that access the medium in an unpredictable fashion. In this case, traditional grant-based policies are highly inefficient or non-applicable, due to the overhead cost and complexity required to coordinate resource sharing, and random access strategies based on variations of ALOHA represent a common solution, e.g. [1], [2]. Gathering a thorough understanding of the behavior of such policies in terms of information freshness represents thus a key step towards the proper design of next-generation IoT systems.

Taking the lead from this remark, we discuss in this paper some recent results in the field of information freshness in random access channels with special attention to IoT setups. To this aim, after a brief introduction to the notion of AoI, we start by reviewing the performance of basic random access policies, namely ALOHA and listen-before-talk, pinpointing some key trade-offs. We then delve into the possibility to achieve significant improvements by leveraging feedback to adapt the channel access probability. Specifically, the notion of age-threshold ALOHA is explored, showing how having terminals transmit only when their AoI exceeds a critical value can yield better levels of information freshness with little impact on throughput. This idea has recently been explored in a number of works, capturing both some fundamental limits and practical algorithms to reach them, e.g. [7]-[9]. In addition, we offer a perspective on a different class of random access policies, which lean on coding and successive interference cancellation to achieve spectral efficiencies comparable to those of their coordinated counterparts, e.g. [10], [11]. When studied from an information freshness standpoint, in fact, these solutions reveal a remarkable potential together with some specific trade-offs that have to be accounted for [12], [13]. Finally, with the aim to stimulate further research and advancements on the topic, we elaborate on some open issues and research challenges that still have to be tackled to reach a mature understanding of information freshness in IoT setups. 


\section{INTRODUCTION TO AOI}

Age of information (AoI) has been proposed and used as a measure of the "staleness" of an information flow at the point where this information is processed. Originally introduced in [5], [14], it is a rather simple-minded notion of staleness, as it only focuses on the time stamp of the packets belonging to the flow, without regard to the information content. Specifically, suppose that for a sequence of samples $\{i=1,2, \ldots\}$, the $i^{t h}$ sample of a source process is generated at time $t_{i}$ and successfully received by the sink at time $t_{i}^{\prime}$. The age $\Delta(t)$ of of the flow at the sink at time $t$ is defined as

$$
\Delta(t)=t-\max \left\{t_{i} \mid t_{i}^{\prime}<t\right\}
$$

and leads to the well-known saw-tooth profile depicted in Fig. 1, where the metric grows linearly with time until being reset upon reception of an update.

In words, the age at time $t$ is the amount of time that elapsed since the newest sample available at the sink was generated at the source. This simple definition deems it very amenable to mathematical analysis. Yet, it has also been shown to be powerful enough to capture performance in a variety of important IoT and cyber-physical system scenarios. For example, in a tracking problem, the deviation in position can be a linear function of age. Moreover, in many control systems (see, e.g. [15]) the mean square error in state estimation increases monotonically with age. Therefore, optimizing AoI has been observed to be a viable method for controlling performance in a large family of systems.

Most works focus on the time-average of AoI, here denoted as $\Delta$, or on statistics of the peak AoI, i.e. the age value right before an update is received. The literature has, however, also provided mechanisms for controlling more elaborate penalty functions of age [16], [17], which in turn allows capturing an even broader range of settings.

The properties of this new performance indicator have piqued the interest of the networking research community not only because of its potential impact on IoT and control systems, but also because of its mathematical richness. AoI miniziation calls indeed for different service policies than traditional delay or throughput optimizations. For example, a LCFS service policy is preferable to FCFS for age optimal operation in a single server queuing system. Moreover, controlling age is distinctly different from either reducing packet delay or increasing overall throughput, as it rather requires a combination of both compoents. Multiple access introduces even richer problems, some of which we will allude to in the rest of this paper.

\section{Age Optimization in ALOHA And CSMA}

A majority of the studies on age-aware random access to date have focused on slotted ALOHA variants. These are deemed particularly suitable for large-scale IoT setups with a large number of participating nodes, each generating data in small amounts and sporadically.

In slotted ALOHA, sources that wish to transmit data attempt transmission at any given time slot with a certain probability. The first analysis of age in slotted ALOHA was
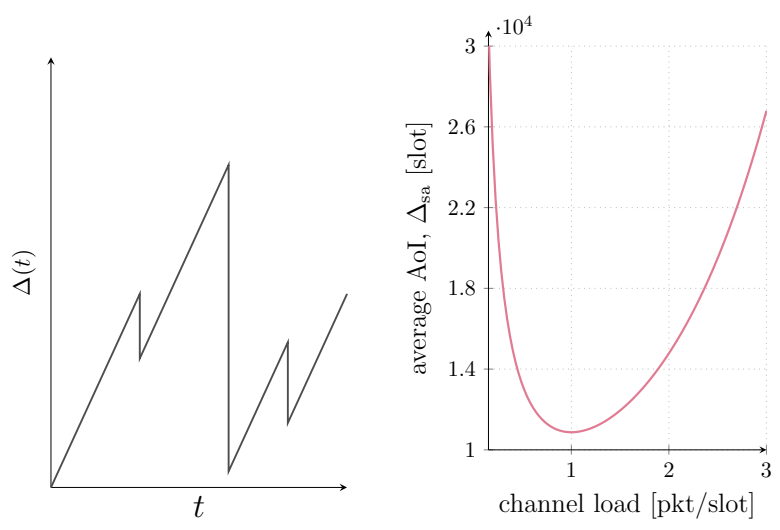

Fig. 1. Leftmost plot: time evolution of AoI. Rightmost plot: average AoI vs channel load for a simple slotted ALOHA policy, for a node population of $\mathrm{n}=4000$ nodes (see Sec. II).

reported in [18], which included an age-optimal selection of the transmission parameter in the absence of feedback and retransmissions. Interestingly, the study revealed how the average AoI exhibits in symmetric conditions an inverse proportionality to the aggregate throughput $\mathrm{S}$, taking the form

$$
\Delta_{\mathrm{sa}}=\frac{1}{2}+\frac{\mathrm{n}}{\mathrm{S}}
$$

where $\mathrm{n}$ is the network population. The well-known trend is reported in the rightmost plot of Fig. 1, clarifying the nonmonotonic behaviour of AoI with respect to channel load. Notably, high AoI values are obtained both with too sporadic transmissions (low channel load) as well as in excessively congested conditions. Analysis of the basic ALOHA procedures was later also extended to the unslotted case in [19], confirming similar trends, and highlighting an even more detrimental effect of collisions on AoI in the fully asynchronous case.

To go beyond these intrinsic limitations, a threshold-based modification of slotted ALOHA was proposed in [20]. This policy, called "Threshold ALOHA (TA)" activates transmission attempts with probability $\tau$ only when a user's age reaches a certain threshold $\gamma$. The parameters $\tau$ and $\gamma$ were jointly optimized in [8]. Note that this policy relies on the availability of feedback to the sender upon a successful transmission, which the sender then uses to update its knowledge about age. The benefit of this one bit feedback per successful transmission was shown to be significant in [8]: the average AoI scales with the network size $n$ as $1.4169 n$, which is a $48 \%$ improvement upon achievable age scaling en with slotted ALOHA. This age reduction is achieved with no significant loss in throughput. Moreover, with improved versions of the TA policy, it is possible to achieve a reduction as large as $79 \%$ [9], which is remarkably close to the theoretically best possible roundrobin limit of $81 \%$ improvement over slotted ALOHA. Similar performance gains are achieved by the SAT policy in [7], which is a distributed policy with a transmission threshold that is set dynamically in response to the estimated number of users in the network.

To complement our overview on random access channels, we note that interesting results have also been recently derived 

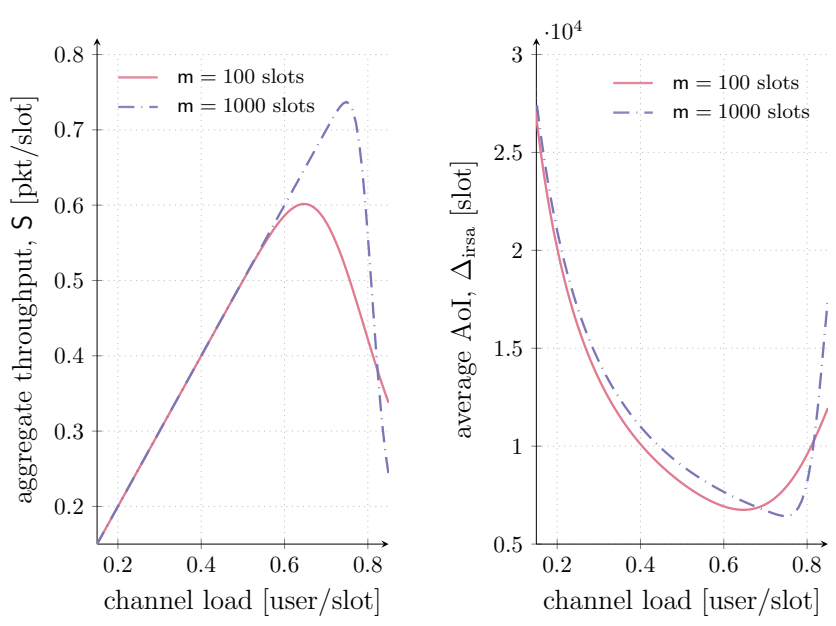

Fig. 2. Aggregate throughput and average AoI vs channel load for IRSA. The protocol operates with a regular distribution, i.e., all nodes send 3 copies of their packets, over frames of duration 100 and 1000 slots. A population of $n=4000$ nodes is considered.

for AoI in carrier sense multiple access (CSMA) settings [21], [22]. Two alternative cases were analyzed in [21], considering the use of CSMA to send (a) stochastically arriving data streams, or (b) packets generated at will, leading to average age expressions in both cases. These are used to optimize back-off time, which, perhaps surprisingly, is found to be equal in both the generate-at-will and exogenous arrival cases.

\section{Modern RAndom ACCESS}

The quest to provide massive connectivity in IoT settings has recently spurred the design of novel uncoordinated medium access policies, aiming to go beyond the intrinsic limitations of the collision-prone ALOHA paradigm. This research line has led to the definition of a number of novel protocols, often dubbed as modern random access, which constructively embrace interference to achieve performance comparable to that of grant-based solutions.

Among them, a pioneering role was played by irregular repetition slotted ALOHA (IRSA) and its evolutions [23]. In such schemes, time is organized in frames composed of an equal number of slots. Any active terminal can access the channel by transmitting a number of replicas of its packet (or coded versions thereof [10]), uniformly placed at random over the slots that compose the next upcoming frame. The number of replicas is in turn drawn from a probability distribution common to all nodes. At the receiver side, processing starts at the end of a frame by looking for singleton (and decodable) slots. Any time a packet is retrived, the signal contribution of its twin copies is removed, possibly leading to other collision-free slots. The potential of this successive interference cancellation (SIC) approach can be fully reaped by optimizing the distribution of the number of sent copies, enabling low packet loss rates and high spectral efficiencies even as the channel occupation increases. In particular, the

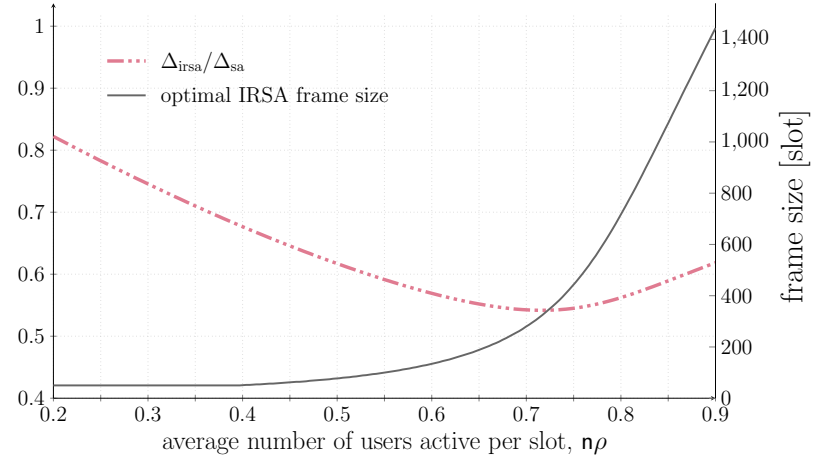

Fig. 3. IRSA frame lenght minimizing average AoI (solid curve, referred to right $y$ axis), and ratio of average AoI of IRSA operated over such frame length and of slotted ALOHA (dashed curve, referred to left $y$ axis). Both quantities are reported against a measure of the channel load, and for a regular IRSA distribution (each node sends three copies of its packet over the frame). A population of $n=4000$ nodes is considered. For further details, see [12].

ideal throughput of 1 [pkt/slot $]^{1}$, characteristic of grant-based access when coordination overhead is disregarded, has been shown to be approachable for asymptotically long frames. On the other hand, shorter frames increase the probability for SIC not to resolve all collisions, and result in lower throughput values, as exemplified in the leftmost plot of Fig. 2.

Interestingly, this fundamental design principle no longer holds when considering information freshness metrics. To clarify the aspect, consider a typical IoT setting in which each device of a large population $\mathrm{n}$ produces an update at every slot with probability $\rho \ll 1$. Assuming a node to access the channel over the next frame to deliver only the freshest generated packet (if any), without receiving any feedback from the destination nor performing any retransmission, a simple yet tight approximation for the average AoI is given by ([12])

$$
\Delta_{\text {irsa }} \simeq \frac{1}{2}+\frac{\mathrm{n}}{\mathrm{S}}+\mathrm{m}
$$

where $m$ is the frame size over which IRSA operates and $\mathrm{S}$ is the aggregate throughput. The reported formulation highlights a key trade-off, specific to information freshness, triggered by m. Indeed, while longer frames can lower AoI by boosting the throughput, they also have a detrimental effect, as i) a new update has to wait on average longer prior to being sent over the next frame, and ii) decoding procedures start only once the whole frame has been buffered. The trend is apparent already in the rightmost plot of Fig. 2, reporting the average AoI for IRSA against channel load for different values of $m$, and is further highlighted in Fig. 3. Specifically, while for low traffic levels shorter frames are to be preferred, under harsh contention the AoI improvement brought by higher throughput values more than outweighs the linear penalty introduced by longer frames. It shall also be stressed that the protocol significantly outperforms slotted ALOHA, without relying on any feedback channel. This is apparent in Fig. 3, where the average AoI can be halved resorting to IRSA in moderately

\footnotetext{
${ }^{1}$ All the results discussed in this section refer to a channel model without erasures, in which singleton slots are always decoded, whereas information cannot be extracted from a slot which sees (possibly after SIC) the superposition of two or more packets.
} 
congested channel conditions. Such a remark confirms the potential of modern random access approaches in terms of information freshness, and points at their optimization as a relevant research for future research, e.g, by tuning the replica transmission distribution based on AoI or by implementing age-threshold adaptation policies.

The relevant role played in an AoI perspective by design criteria that go beyond throughput maximization has recently emerged also for other advanced random access schemes. This is the case, for example, of frameless ALOHA [24], whose working principle was inspired by an analogy with rateless codes. Targeting massive IoT settings, the protocol once more leans on SIC performed across multiple packet copies, yet, in contrast to IRSA, does not enforce frames of fixed duration. Channel contention phases are initiated by a beacon, broadcast by the gateway that is collecting information. After this, all nodes with data to transmit access the medium at each successive slot with a given probability, sending copies of their data unit. Each time a packet is decoded (i.e., received without collision), interference cancellation is performed across all other slots in which it is sent. The contention continues until a target number of users have been decoded or a maximum frame duration is reached. This scheme is known to grant good throughput performance, and its AoI behavior was first captured in [13], highlighting two key takeaways. On the one hand, the ability to tune the frame duration to the number of contending users can indeed be highly beneficial when information freshness is targeted. On the other hand, driving the system towards an AoI minimization may entail losses in throughput. A thorough understanding of such trade-offs is thus paramount for the design and proper operation of IoT communications in different settings.

From this standpoint, we conclude our discussion by pointing out how a parallel and relevant research direction lies in the study of different families of modern random access protocols that have recently been proposed. Particularly interesting are a number of solutions inspired by the seminal work of Polyanskiy [25]. Rather than having nodes send multiple copies of their packets, these schemes jointly tackle medium access and channel coding, allowing terminals to share a common codebook, and employing techniques such as compressive sensing to recover the set of transmissions [26], [27]. Following this approach, dramatic improvements have been shown towards the efficient support of a massive number of terminals, rendering this paradigm a strong candidate for next-generation IoT systems. Nonetheless, their behaviour from an information freshness standpoint remains unexplored, posing an important open challenge.

\section{OPEn Challenges AND RESEARCH DiRECTIONS}

\section{A. Age-adaptive extensions of conventional random access} schemes

Most of the analyses on slotted ALOHA variants have to date been done under symmetry assumptions for users of the network and for information streams. Moreover, some of these policies achieved the described age and throughput performance under the assumption of ideal feedback. Hence, broader principles for the optimal design of age-threshold based policies to work robustly under realistic network and traffic conditions in a distributed manner are waiting to be developed.

\section{B. Newer AoI-related metrics}

AoI-centered optimizations have often focused on keeping a low time average age or low peak age across all time, without regard to the points at which the information updates will be used. The broad notion of the design of a communication system (from the sampling or generation of the data to its encoding and transport) to account for the purpose and value of the information to be transmitted has been broadly defined, perhaps with an abuse of terminology, as semantic communication [28]. Various metrics, in addition to AoI, have been suggested to be useful under the umbrella of semantic communication, such as age of incorrect information (AoII) [29], value of information [30], and query age of information (QAoI) [31].

AoII measures how much the data at the destination has aged since the most recent change on the source side. Hence, this metric stays constant as long as the data measured on the source side has not changed, and accounts for a penalty whenever the sink has an outdated perception of the status of the monitored process. This is particularly critical for scenarios (e.g., cyberphysical systems) where an actuation will take place on the destination side based on the current data sample. AoII, studied in [29] in a point-to-point scenario, provides an interesting tool to go beyond a fundamental limitation of AoI and track the staleness of the information content rather than of its time stamps. In this perspective, the exploration of the metric (or variations thereof) in random access settings, together with the definition of joint sampling and transmission policies that account for the nature of the processes being tracked remain open and very promising areas for research.

QAoI [31] measures the freshness of the information at the query instants, that is, at points in time when the destination generates a query requiring the new update. A query source is typically an application that requests information updates from a remote source, to use these updates in a computation or actuation. This metric is thus well suited especially to pull-based communication scenarios, where, in contrast to the traditional push based network model, transmissions are triggered not by the source but by the destination. Recently, metrics similar to QAoI have been studied in the literature, some of which motivated by analytical convenience. This is the case, for instance, of effective AoI (EAoI), defined and analyzed on a discrete time model in [32], which is set equal to AoI at time frames containing a query, and set to zero in others. The metric provides a lower bound to QAoI.

One of the essentially interesting instances of the pull-based AoI minimization problem is when query instants are not known by the transmitting source. If queries are knowm ahead of time (e.g. they are deterministic) or known instantaneously (or with a small delay), the source could generate updates to satisfy these queries, achieving an arbitrarily low QAoI. If this anti-causal or instantaneous information about the query 
generation process is not available, but feedback about past queries are available (in the form of success feedback from the destination, for example), the source may use this information to infer the state of the QAoI, and in some cases the state of the Query Process, and use this as an input to its transmission policy. The more interesting case is when the query process is not even causally known, but certain statistics of the query process is available to the source. It is an open problem to design random access policies with the goal of achieving low QAoI under this no feedback assumption. Recently, [33] developed optimal packet generation policies for a single node, in the no feedback model.

The WiFresh application proposed in [34] is a recent proof of concept of how powerful a polling based approach can be to optimize freshness in a Wi-Fi environment. WiFresh uses a combination of polling from the base station, MaxWeight (MW) scheduling and LCFS queues at the application layer. Unlike age-aware flow control mechanisms that adopt the "generate-at-will" model, such as ACP [35], WiFresh does not attempt to control the packet generation rate at the sources. Therefore, it can potentially get overloaded when the number of sources increases. However, under moderate load, it has been show to reduce aging in the network by an order of magnitude or more, with respect to the conventional approach of keeping FCFS queues with UDP over Wi-Fi. This result hints at two effective shifts in the design of future multiple access strategies geared toward freshness: adopting pull-based or polling based mechanisms at the link layer that cooperate with the application layer at sources, and keeping LCFS queues or combining the application layer sampling and data generation with the MAC-layer service policy at senders. As both approaches go against mainstream networking practice, they contain many open research problems.

\section{REFERENCES}

[1] LoRa Alliance Technical Commitee, "LoRaWAN 1.1 Specification."

[2] Sigfox, "SIGFOX: The Global Communications Service Provider for the Internet of Things," www.sigfox.com.

[3] Y. Wang, X. Lin, A. Adhikary, A. Grovlen, Y. Sui, Y. Blankenship, J. Bergman, and H. Razaghi, "A primer on 3GPP narrowband internet of things," IEEE Commun. Mag., vol. 55, no. 3, pp. 117-123, 2017.

[4] "Study on narrow-band Internet of things (NB-IoT) / enhanced machine type communication (eMTC) support for non-terrestrial networks (NTN)," 3GPP TR 36.763, Jun. 2021.

[5] S. Kaul, R. Yates, and M. Gruteser, "Real-time status: How often should one update?" in Proc. IEEE INFOCOM, 2012.

[6] R. D. Yates, Y. Sun, D. R. Brown, S. K. Kaul, E. Modiano, and S. Ulukus, "Age of information: An introduction and survey," IEEE $J$. Sel. Areas Commun., vol. 39, no. 5, pp. 1183-1210, 2021.

[7] X. Chen, K. Gatsis, H. Hassani, and S. Bidokhti, "Age of information in random access channels," in Proc. IEEE ISIT, 2020.

[8] O. T. Yavascan and E. Uysal, "Analysis of slotted ALOHA with an age threshold," IEEE J. Sel. Areas Commun., vol. 39, no. 5, pp. 1456-1470, May 2021.

[9] M. Ahmetoglu, O. Yavascan, and E. Uysal, "Mista: an ageoptimized slotted ALOHA protocol," 2021. [Online]. Available: http://arxiv.org/abs/2105.05129

[10] E. Paolini, G. Liva, and M. Chiani, "Coded slotted ALOHA: A graphbased method for uncoordinated multiple access," IEEE Trans. Inf. Theory, vol. 61, no. 12, pp. 6815-6832, 2015.

[11] F. Lazaro, C. Stefanovic, and P. Popovski, "Reliability-latency performance of frameless ALOHA with and without feedback," IEEE Transactions on Communications, 2020.
[12] A. Munari, "Modern random access: an age of information perspective on irregular repetition slotted ALOHA," IEEE Trans. Commun., vol. 69, no. 6, pp. 3572-3585, Jun. 2021.

[13] A. Munari, F. Lazaro, G. Liva, and G. Durisi, "An age of information characterization of frameless aloha," in Proc. IEEE ASILOMAR, 2021.

[14] S. Kaul, M. Gruteser, V. Rai, and J. Kenney, "Minimizing age of information in vehicular networks," in Proc. IEEE SECON, 2011.

[15] O. Ayan, M. Vilgelm, M. Klügel, S. Hirche, and W. Kellerer, "Age-ofinformation vs. value-of-information scheduling for cellular networked control systems," in Proc. ACM/IEEE International Conference on Cyber-Physical Systems, 2019.

[16] Y. Sun, E. Uysal-Biyikoglu, R. Yates, C. E. Koksal, and N. B. Shroff, "Update or wait: How to keep your data fresh," in Proc. IEEE INFOCOM, 2016

[17] A. Munari and G. Liva, "Information freshness analysis of slotted ALOHA in Gilbert-Elliot channels," IEEE Commun. Lett., 2021.

[18] R. Yates and S. K. Kaul, "Status updates over unreliable multiaccess channels," in Proc. IEEE ISIT, 2017.

[19] R. Yates and S. Kaul, "Age of information in uncoordinated unslotted updating," in Proc. IEEE ISIT, 2020.

[20] D. C. Atabay, E. Uysal, and O. Kaya, "Improving age of information in random access channels," in Proc. IEEE INFOCOM Workshops, 2020.

[21] A. Maatouk, M. Assaad, and A. Ephremides, "On the age of information in a csma environment," IEEE/ACM Transactions on Networking, vol. 28 , no. 2 , pp. 818-831, 2020.

[22] A. Baiocchi and I. Turcanu, "Age of information of one-hop broadcast communications in a csma network," IEEE Communications Letters, vol. 25, no. 1, pp. 294-298, 2021.

[23] G. Liva, "Graph-based analysis and optimization of contention resolution diversity slotted ALOHA," IEEE Trans. Commun., vol. 59, no. 2, pp. 477-487, 2011.

[24] C. Stefanovic and P. Popovski, "Aloha random access that operates as a rateless code," IEEE Trans. Commun., vol. 61, no. 11, pp. 4653-4662, 2013.

[25] Y. Polyanskiy, "A perspective on massive random-access," in Proc. IEEE ISIT, 2017.

[26] V. K. Amalladinne, J. F. Chamberland, and K. R. Narayanan, "A coded compressed sensing scheme for unsourced multiple access," IEEE Trans. Inf. Theory, vol. 66, no. 10, pp. 6509-6533, 2020.

[27] A. Fengler, P. Jung, and G. Caire, "Sparcs for unsourced random access," IEEE Trans. Inf. Theory, 2021.

[28] E. Uysal, O. Kaya, A. Ephremides, J. Gross, M. Codreanu, P. Popovski, M. Assaad, G. Liva, A. Munari, T. Soleymani, B. Soret, and K. H Johansson, "Semantic communications in networked systems," 2021.

[29] A. Maatouk, S. Kriouile, M. Assaad, and A. Ephremides, "The age of incorrect information: A new performance metric for status updates," IEEE/ACM Transactions on Networking, vol. 28, no. 5, pp. 2215-2228, 2020.

[30] T. Soleymani, J. S. Baras, and S. Hirche, "Value of information in feedback control," 2018.

[31] F. Chiariotti, J. Holm, A. E. Kalør, B. Soret, S. K. Jensen, T. B. Pedersen, and P. Popovski, "Query age of information: Freshness in pull-based communication," CoRR, vol. abs/2105.06845, 2021. [Online]. Available: https://arxiv.org/abs/2105.06845

[32] B. Yin, S. Zhang, Y. Cheng, L. X. Cai, Z. Jiang, S. Zhou, and Z. Niu, "Only those requested count: Proactive scheduling policies for minimizing effective age-of-information," in IEEE INFOCOM 2019 IEEE Conference on Computer Communications, 2019, pp. 109-117.

[33] E. Ildiz, O. T. Yavascan, O. T. Kartal, and E. Uysal, "Pull or wait: How to optimize query age of information," CoRR, 2021.

[34] I. Kadota, M. S. Rahman, and E. Modiano, "Wifresh: Age-ofinformation from theory to implementation," 2020.

[35] T. Shreedhar, S. K. Kaul, and R. D. Yates, "An age control transport protocol for delivering fresh updates in the internet-of-things," in 2019 IEEE 20th International Symposium on "A World of Wireless, Mobile and Multimedia Networks" (WoWMoM), 2019, pp. 1-7. 